\title{
European Option Pricing for a Stochastic Volatility Lévy Model with Stochastic Interest Rates
}

\author{
Sarisa Pinkham, Pairote Sattayatham \\ School of Mathematics, Institute of Science, Suranaree University of Technology, Nakhon Ratchasima, Thailand \\ E-mail:sarisa@math.sut.ac.th,pairote@sut.ac.th \\ Received August 27, 2011; revised September 9, 2011; accepted September 18, 2011
}

\begin{abstract}
We present a European option pricing when the underlying asset price dynamics is governed by a linear combination of the time-change Lévy process and a stochastic interest rate which follows the Vasicek process. We obtain an explicit formula for the European call option in term of the characteristic function of the tail probabilities.
\end{abstract}

Keywords: Time-Change Lévy Process, Stochastic Interest Rate, Vasicek Process, Forward Measure, Option Pricing

\section{Introduction}

Let $(\Omega, F, P)$ be a probability space. A stochastic process $L_{t}$ is a Lévy process if it has independent and stationary increments and has a stochastically continuous sample path, i.e. for any $\varepsilon>0, \lim _{h \downarrow 0} P\left(\left|L_{t+h}-L_{t}\right|>\varepsilon\right) \rightarrow 0$. The simplest possible Lévy processes are the standard Brownian motion $W_{t}$, Poisson process $N_{t}$, and compound Poisson process $\sum_{i=1}^{N_{t}} Y_{i}$ where $N_{t}$ is Poisson process with intensity $\lambda t$ and $Y_{i}$ are i.i.d. random variables. Of course, we can build a new Lévy process from known ones by using the technique of linear transformation. For example, the jump diffusion process $\mu t+\sigma W_{t}+\sum_{i=1}^{N_{t}} Y_{i}$, where $\mu, \sigma$ are constants, is a Lévy process which comes from a linear transformation of two independent Lévy processes, i.e. a Brownian motion with drift and a compound Poisson process.

Assume that a risk-neutral probability measure $Q$ exists and all processes in Section 1 will be considered under this risk-neutral measure.

In the Black-Scholes model, the price of a risky asset $S_{t}$ under a risk-neutral measure $Q$ and with non dividend payment follows

$$
S_{t}=S_{0} \exp \left(\tilde{L}_{t}\right)=S_{0} \exp \left(r t+\left(\sigma W_{t}-\frac{1}{2} \sigma^{2} t\right)\right)
$$

where $r \in \mathbb{R}$ is a risk-free interest rates, $\sigma \in \mathbb{R}$ is a vo- latility coefficient of the stock price. Instead of modeling the $\log$ returns

$$
\tilde{L}_{t}=r t+\left(\sigma W_{t}-\frac{1}{2} \sigma^{2} t\right)
$$

with a normal distribution. We now replace it with a more sophisticated process $L_{t}$ which is a Lévy process of the form

$$
L_{t}=r t+\left(\sigma W_{t}-\frac{1}{2} \sigma^{2} t\right)+\left(J_{t}-\zeta t\right),
$$

where $J_{t}$ and $\zeta_{t}$ denotes a pure Lévy jump component, (i.e. a Lévy process with no Brownian motion part) and its convexity adjustment. We assume that the processes $W_{t}$ and $J_{t}$ are independent. To incorporate the volatileity effect to the model (1.2), we follow the technique of Carr and $\mathrm{Wu}$ [1] by subordinating a part of a standard Brownian motion $\sigma W_{t}-\frac{1}{2} \sigma^{2} t$ and a part of jump Lévy process $J_{t}-\zeta t$ by the time integral of a mean reverting Cox Ingersoll Ross (CIR) process

$$
T_{t}=\int_{0}^{t} v_{s} \mathrm{~d} s,
$$

where $v_{t}$ follows the CIR process

$$
\mathrm{d} v_{t}=\gamma\left(1-v_{t}\right) \mathrm{d} t+\sigma_{v} \sqrt{v_{t}} \mathrm{~d} W_{t}^{v}
$$

Here $W_{t}^{v}$ is a standard Brownian motion which corresponds to the process $v_{t}$. The constant $\gamma \in \mathbb{R}$ is the rate at which the process $v_{t}$ reverts toward its long term mean and $\sigma_{v}>0$ is the volatility coefficient of the process $v_{t}$. 
Hence, the model (1.2) has been changed to

$$
L_{t}=r t+\left(\sigma W_{T_{t}}-\frac{1}{2} \sigma^{2} T_{t}\right)+\left(J_{T_{t}}-\zeta T_{t}\right),
$$

and this new process is called a stochastic volatility Levy process. One can interpret $T_{t}$ as the stochastic clock process with activity rate process $v_{t}$. By replacing $\tilde{L}_{t}$ in (1.1) with $L_{t}$, we obtain a model of an underlying asset under the risk-neutral measure $Q$ with stochastic volatility as follows:

$$
S_{t}=S_{0} \exp \left(r t+\left(\sigma W_{T_{t}}-\frac{1}{2} \sigma^{2} T_{t}\right)+\left(J_{T_{t}}-\zeta T_{t}\right)\right)(1.5)
$$

In this paper, we shall consider the problem of finding a formula for European call options based on the underlying asset model (1.5) for which the constant interest rates $r$ is replaced by the stochastic interest rates $r_{t}$, and $J_{t}$ is compound Poisson process, i.e. the model under our consideration is given by

$$
S_{t}=S_{0} \exp \left(r_{t} t+\left(\sigma W_{T_{t}}-\frac{1}{2} \sigma^{2} T_{t}\right)+\left(J_{T_{t}}-\zeta T_{t}\right)\right)
$$

Here, we assume that $r_{t}$ follows the Vasicek process

$$
\mathrm{d} r_{t}=\left(\alpha-\beta r_{t}\right) \mathrm{d} t+\sigma_{r} \mathrm{~d} W_{t}^{r},
$$

$W_{t}^{r}$ is a standard Brownian motion with respect to the process $r_{t}$ and $\mathrm{d} W_{t}^{r} \mathrm{~d} W_{t}^{v}=\mathrm{d} W_{t}^{r} \mathrm{~d} W_{t}=0$. The constant $\beta>0$ is the rate at which the interest rate reverts toward its long term mean, $\sigma_{r}>0$ is the volatility coefficient of the interest rate process (1.7), The constant $\alpha>0$ is a speed reversion.

\section{Literature Reviews}

Many financial engineering studies have been undertaken to modify and improve the Black-Scholes model. For example, The jump diffusion models of Merton [2], the stochastic Volatility jump diffusion model of Bates [3] and Yan and Hanson [4]. Furthermore, the time change Lévy models proposed by Carr and $\mathrm{Wu}[1]$.

The problem of option pricing under stochastic interest rates has been investigated for along time. Kim [5] constructed the option pricing formula based on Black-Scholes model under several stochastic interest rate processes, i.e., Vasicek, CIR, Ho-Lee type. He found that by incurporating stochastic interest rates into the Black-Scholes model, for a short maturity option, does not contribute to improvement in the performance of the original BlackScholes' pricing formula. Brigo and Mercurio [6] mention that the stochastic feature of interest rates has a stronger impact on the option price when pricing for a long maturity option. Carr and $\mathrm{Wu}[1]$ continue this study by giving the option pricing formula based on a time-changed Lévy process model. But they still use constant interest rates in the model.

In this paper, we give an analysis on the option pricing model based on a time-changed Lévy process with stochastic interest rates.

The rest of the paper is organized as follows. The dynamics under the forward measure is described in Section 3. The option pricing formula is given in Section 4. Finally, the close form solution for a European call option in terms of the characteristic function is given in Section 5.

\section{The Ddynamics under the Forward Measure}

We begin by giving a brief review of the definition of a correlated Brownian motion and some of its properties (for more details one see Brummelhuis [7]). Recalling that a standard Brownian motion in $R^{n}$ is a stochastic process $\left(Z_{t}\right)_{t \geq 0}$ whose value at time $t$ is simply a vector of $n$ independent Brownian motions at $t$,

$$
Z_{t}=\left(Z_{1, t}, \cdots, Z_{n, t}\right)
$$

We use $Z$ instead of $W$ since we would like to reserve the latter for the more general case of correlated Brownian motion, which will be defined as follows:

Let $\rho=\left(\rho_{i j}\right)_{1 \leq i, j \leq n}$ be a (constant) positive symmetric matrix satisfying $\rho_{i i}=1$ and $-1 \leq \rho_{i j} \leq 1$ By Cholesky's decomposition theorem, one can find an upper triangul $n \times n$ matrix $H=\left(h_{i j}\right)$ such that $\rho=H H^{t}$, where

$H^{t}$ is the transpose of the matrix $H$. Let

$Z_{t}=\left(Z_{1, t}, \cdots, Z_{n, t}\right)$ be a standard Brownian motion as introduced above, we define a new vector-valued process $\boldsymbol{W}_{t}=\left(W_{1, t}, \cdots, W_{n, t}\right)$ by $\boldsymbol{W}_{t}=H \mathbf{Z}_{t}$ or in term of components,

$$
W_{i, t}=\sum_{j=1}^{n} h_{i j} Z_{j, t}, i=1, \cdots, n
$$

The process $\left(\boldsymbol{W}_{t}\right)_{t \geq 0}$ is called a correlated Brownian motion with a (constant) correlation matrix $\rho$. Each component process $\left(W_{i, t}\right)_{t \geq 0}$ is itself a standard Brownian motion. Note that if $\rho=I d$ (the identity matrix) then $W_{t}$ is a standard Brownian motion. For example, if we let a symmetric matrix

$$
\rho=\left[\begin{array}{ccc}
1 & \rho_{v} & 0 \\
\rho_{v} & 1 & 0 \\
0 & 0 & 1
\end{array}\right]
$$

Then $\rho$ has a Cholesky decomposition of the form $\rho=H H^{T}$ where $H$ is an upper triangular matrix of the form 


$$
H=\left[\begin{array}{ccc}
\sqrt{1-\rho_{v}^{2}} & \rho_{v} & 0 \\
0 & 1 & 0 \\
0 & 0 & 1
\end{array}\right]
$$

Let $Z_{t}=\left(Z_{t}, Z_{t}^{r}, Z_{t}^{v}\right)$ be three independent Brownian motions then $\boldsymbol{W}_{t}=\left(W_{t}, W_{t}^{r}, W_{t}^{v}\right)$ defined by $\boldsymbol{W}_{t}=\mathrm{H} \boldsymbol{Z}_{t}$, or in terms of components,

$$
W_{t}=\left(\sqrt{1-\rho_{v}^{2}}\right) Z_{t}+\rho_{v} Z_{t}^{v}, W_{t}^{v}=Z_{t}^{v}, W_{t}^{r}=Z_{t}^{r}
$$

Now let us turn to our problem. Note that, by Ito's lemma, the model (1.6) has the dynamic given by

$$
\begin{aligned}
& \mathrm{d} S_{t}=S_{t}\left(\left(r_{t}-\lambda_{m} v_{t}\right) \mathrm{d} t+\sigma \mathrm{d} W_{T_{t}}\right)+S_{t-}\left(\mathrm{e}^{Y_{t}}-1\right) \mathrm{d} N_{T_{t}}, \\
& \mathrm{~d} r_{t}=\left(\alpha-\beta r_{t}\right) \mathrm{d} t+\sigma_{r} \mathrm{~d} W_{t}^{r}, \\
& \mathrm{~d} v_{t}=\gamma\left(1-v_{t}\right) \mathrm{d} t+\sigma_{v} \sqrt{v_{t}} \mathrm{~d} W_{t}^{v},
\end{aligned}
$$

where $\lambda_{m}=\lambda E\left(\mathrm{e}^{Y_{t}}-1\right), \quad \mathrm{d} W_{t} \mathrm{~d} W_{t}^{r}=\mathrm{d} W_{t} \mathrm{~d} W_{t}^{r}=0$ and $\mathrm{d} W_{t} \mathrm{~d} W_{t}^{v}=\rho_{v} \mathrm{~d} t$.

We can re-write the dynamic (3.3) in terms of three independent Brownian motions $\left(Z_{t}, Z_{t}^{v}, Z_{t}^{r}\right)$ follows (3.2), we get

$$
\begin{gathered}
\mathrm{d} S_{t}=S_{t}\left(\left(r_{t}-\lambda_{m} v_{t}\right) \mathrm{d} t+\sigma \sqrt{v_{t}}\left(\rho_{v} d Z_{t}^{v}+\sqrt{1-\rho_{v}^{2}} \mathrm{~d} Z_{t}\right)\right) \\
+S_{t-}\left(\mathrm{e}^{Y_{t}}-1\right) \mathrm{d} N_{T_{t}}, \\
\mathrm{~d} r_{t}=\left(\alpha-\beta r_{t}\right) \mathrm{d} t+\sigma_{r} \mathrm{~d} Z_{t}^{r}, \\
\mathrm{~d} v_{t}=\gamma\left(1-v_{t}\right) \mathrm{d} t+\sigma_{v} \sqrt{v_{t}} \mathrm{~d} Z_{t}^{v},
\end{gathered}
$$

This decomposition makes it easier to perform a measure transformation. In fact, for any fixed maturity $T$, let us denote by $Q^{T}$ the $T$-forward measure, i.e. the probability measure that is defined by the RadonNikodym derivative,

$$
\frac{\mathrm{d} Q^{T}}{\mathrm{~d} Q}=\frac{\exp \left(-\int_{0}^{T} r_{u} \mathrm{~d} u\right)}{P(0, T)}
$$

Here, $P(t, T)$ is the price at time $t$ of a zero-coupon bond with maturity $T$ and is defined as

$$
P(t, T)=E_{Q}\left[\mathrm{e}^{-\int_{t}^{T} r_{s} d s} \mid F_{t}\right]
$$

Next, Consider a continuous-time economy where interest rates are stochastic and satisfy (3.5). Since the SDE (3.5) satisfies all the necessary conditions of Theorem 32, see Protter [8], then the solution of (3.5) has the Markov property. As a consequence, the zero coupon bond price at time $t$ under the measure $Q$ in (3.8) satisfies

$$
P(t, T)=E_{Q}\left[\exp \left(-\int_{t}^{T} r_{s} \mathrm{~d} s\right) \mid r_{t}\right]
$$

Note that $P(t, T)$ depends on $r_{t}$ only instead of depending on all information available in $F_{t}$ up to time $t$. As such, it becomes a function $F\left(t, r_{t}\right)$ of $r_{t}$,

$$
P(t, T)=F\left(t, r_{t}\right),
$$

meaning that the pricing problem can now be formulated as a search for the function $F\left(t, r_{t}\right)$.

Lemma 1 The price of a zero coupon bond can be derived by computing the expectation (3.9). We obtain

$$
P(t, T)=\exp \left(a(t, T)+b(t, T) r_{t}\right)
$$

where

$$
b\left(t, r_{t}\right)=\frac{1}{\beta}\left(\mathrm{e}^{-\beta(T-t)}-1\right),
$$

$$
\begin{aligned}
a(t, T)= & \left(\frac{\alpha}{\beta^{2}}-\frac{3 \sigma_{r}^{2}}{4 \beta^{3}}\right)-\frac{\sigma_{r}^{2}}{4 \beta^{3}} \mathrm{e}^{-2 \beta(T-t)} \\
& +\left(\frac{\sigma_{r}^{2}}{\beta^{3}}-\frac{\alpha}{\beta^{2}}\right) \mathrm{e}^{-\beta(T-t)}+\left(\frac{\sigma_{r}^{2}}{2 \beta^{2}}-\frac{\alpha}{\beta^{2}}\right)(T-t)
\end{aligned}
$$

Proof. See Privault [9] (pp. 38-39).

Lemma 2 The process $r_{t}$ following the dynamics in (3.5) can be written in the form

$$
r_{t}=x_{t}+w(t), \text { for each } t
$$

where the process $x_{t}$ satisfies

$$
\mathrm{d} x_{t}=-\beta x_{t} \mathrm{~d} t+\sigma_{r} \mathrm{~d} Z_{t}^{r}, x_{0}=0 .
$$

Moreover, the function $w(t)$ is deterministic and well defined in the time interval $[0, T]$ which satisfied

$$
w(t)=r_{0} \mathrm{e}^{-\beta t}+\frac{\alpha}{\beta}\left(1-\mathrm{e}^{-\beta t}\right)
$$

In particular, $w(0)=r_{0}$.

Proof. To solve the solution of SDE (3.5),

Let $g(t, r)=e^{\beta t} r$ and using Ito's Lemma

$$
\mathrm{d} g=\frac{\partial g}{\partial t} \mathrm{~d} t+\frac{\partial g}{\partial r} \mathrm{~d} r+\frac{1}{2} \frac{\partial^{2} g}{\partial r^{2}}(\mathrm{~d} r)^{2},
$$

Then,

$$
\begin{aligned}
\mathrm{d} e^{\beta t} r_{t} & =\beta e^{\beta t} r_{t} \mathrm{~d} t+\mathrm{e}^{\beta t}\left(\left(\alpha-\beta r_{t}\right) \mathrm{d} t+\sigma_{r} \mathrm{~d} Z_{t}^{r}\right) \\
& =\alpha e^{\beta t} \mathrm{~d} t+\mathrm{e}^{\beta t} \sigma_{r} \mathrm{~d} Z_{t}^{r}
\end{aligned}
$$

Integrated on both side the above equation from 0 to $t$ where $0<t \leq T$ and simplified, one get

$$
r_{t}=r_{0} \mathrm{e}^{-\beta t}+\frac{\alpha}{\beta}\left(1-\mathrm{e}^{-\beta t}\right)+\sigma_{r} \int_{0}^{t} \mathrm{e}^{-\beta(t-u)} \mathrm{d} Z_{u}^{r}
$$

By using the definition of $w(t)$ form (3.13), 


$$
r_{t}=w(t)+\sigma_{r} \int_{0}^{t} \mathrm{e}^{-\beta(t-u)} \mathrm{d} Z_{u}^{r}
$$

where

$$
w(t)=r_{0} \mathrm{e}^{-\beta t}+\frac{\alpha}{\beta}\left(1-\mathrm{e}^{-\beta t}\right) .
$$

Note that the solution of (3.12) is

$$
x_{t}=x_{0} \mathrm{e}^{-\beta t}+\sigma_{r} \int_{0}^{t} \mathrm{e}^{-\beta(t-u)} \mathrm{d} Z_{u}^{r}=\sigma_{r} \int_{0}^{t} \mathrm{e}^{-\beta(t-u)} \mathrm{d} Z_{u}^{r} .
$$

Hence, $r_{t}=w(t)+x_{t}$ for each $t$. The proof is now complete.

Next we shall calculate the Radon-Nikodym derivative as appear in (3.7). By Lemma 1 and 2, we have $r_{t}=x_{t}+w(t)$ and $P(0, T)$. Substituting $r_{t}$ and $P(0, T)$ into (3.7), we have

$$
\begin{aligned}
\frac{\mathrm{d} Q^{T}}{\mathrm{~d} Q} & =\frac{\exp \left(-\int_{0}^{T} x_{u}+w(u) \mathrm{d} u\right)}{\exp \left(a(0, T)+b(0, T) r_{0}\right)} \\
& =\exp \left(-\int_{0}^{T} x_{u} \mathrm{~d} u-\frac{\sigma^{2}}{2 \beta^{2}} \int_{0}^{T}\left(1-e^{-\beta(T-u)}\right)^{2} \mathrm{~d} u\right)
\end{aligned}
$$

Stochastic integration by parts implies that

$$
\int_{0}^{T} x_{u} \mathrm{~d} u=T x_{T}-\int_{0}^{T} u \mathrm{~d} x_{u}=\int_{0}^{T}(T-u) \mathrm{d} x_{u}
$$

By substituting the expression for $d x_{u}$ from (3.12),

$$
\begin{aligned}
& \int_{0}^{T}(T-u) \mathrm{d} x_{u} \\
= & -\beta \int_{0}^{T}(T-u) x_{u} \mathrm{~d} u+\sigma_{r} \int_{0}^{T}(T-u) \mathrm{d} Z_{u}^{r}
\end{aligned}
$$

Moreover by substituting the expression for $x_{u}$ from (3.16), the first integral on the right hand side of (3.19) becomes

$$
\begin{aligned}
& -\beta \int_{0}^{T}(T-u) x_{u} \mathrm{~d} u \\
= & -\beta \sigma_{r} \int_{0}^{T}\left((T-u) \int_{o}^{u} \mathrm{e}^{-\beta(u-s)} \mathrm{d} Z_{u}^{r}\right) \mathrm{d} u
\end{aligned}
$$

Using integral by parts, we have (Equation 3.21)

Substituting (3.21) into (3.19), we obtain

$$
\int_{0}^{T}(T-u) \mathrm{d} x_{u}=-\frac{\sigma_{r}}{\beta}\left[\int_{0}^{T}\left(\mathrm{e}^{-\beta(T-u)}-1\right) \mathrm{d} Z_{u}^{r}\right]
$$

Hence, $\int_{0}^{T} x_{u} \mathrm{~d} u=-\frac{\sigma_{r}}{\beta}\left[\int_{0}^{T}\left(\mathrm{e}^{-\beta(T-u)}-1\right) \mathrm{d} Z_{u}^{r}\right]$

Substituting (3.22) into (3.17), once get

$$
\begin{aligned}
& \frac{\mathrm{d} Q^{T}}{\mathrm{~d} Q} \\
= & \exp \left(\frac{\sigma_{r}}{\beta} \int_{0}^{T}\left(1-\mathrm{e}^{-\beta(T-u)}\right) \mathrm{d} Z_{u}^{r}-\frac{\sigma_{r}^{2}}{2 \beta^{2}} \int_{0}^{T}\left(1-\mathrm{e}^{-\beta(T-u)}\right)^{2} \mathrm{~d} u\right)
\end{aligned}
$$

The Girsanov theorem then implies that the three processes $Z_{t}^{r T}, Z_{t}^{v T}$ and $Z_{t}^{T}$ defined by

$$
\begin{aligned}
& \mathrm{d} Z_{t}^{r T}=\mathrm{d} Z_{t}^{r}+\frac{\sigma_{r}}{\beta}\left(1-\mathrm{e}^{-\beta(T-t)}\right) \mathrm{d} t \\
& \mathrm{~d} Z_{t}^{v T}=\mathrm{d} Z_{t}^{v}, \mathrm{~d} Z_{t}^{T}=\mathrm{d} Z_{t}
\end{aligned}
$$

are three independent Brownian motions under the measure $Q^{T}$. Therefore, the dynamics of $r_{t}, v_{t}$ and $S_{t}$ under $Q^{T}$ are given by

$$
\begin{aligned}
\mathrm{d} S_{t}= & S_{t}\left(\left(r_{t}-\lambda_{m} v_{t}\right) \mathrm{d} t+\rho_{v} \sigma \sqrt{v_{t}} \mathrm{~d} Z_{t}^{v T}+\sigma \sqrt{v_{t}\left(1-\rho_{v}^{2}\right)} \mathrm{d} Z_{t}^{T}\right) \\
& +S_{t-}\left(\mathrm{e}^{Y_{t}}-1\right) \mathrm{d} N_{T_{t}}, \\
\mathrm{~d} r_{t}= & \left(\alpha-\beta r_{t}-\frac{\sigma_{r}^{2}}{\beta}\left(1-\mathrm{e}^{-\beta(T-t)}\right)\right) \mathrm{d} t+\sigma_{r} \mathrm{~d} Z_{t}^{r T}, \\
\mathrm{~d} v_{t}= & \gamma\left(1-v_{t}\right) \mathrm{d} t+\sigma_{v} \sqrt{v_{t}} \mathrm{~d} Z_{t}^{v T} .
\end{aligned}
$$

\section{The Pricing of a European Call Option on the Given Asset}

Let $\left(S_{t}\right)_{t \in[0, T]}$ be the price of a financial asset modeled as a stochastic process on a filtered probability space $\left(\Omega, F, F_{t}, Q^{T}\right), \quad F_{t}$ is usually taken to be the price history up to time $t$. All processes in this section will be defined in this space. We denote $C$ the price at time $t$ of a European $\mathrm{u}$ call option on the current price of an underlying asset $S_{t}$ with strike price $K$ and expiration time $T$.

$$
\begin{aligned}
& -\beta \sigma_{r} \int_{0}^{T}\left((T-u) \int_{0}^{u} \mathrm{e}^{-\beta(u-s)} \mathrm{d} Z_{u}^{r}\right) \mathrm{d} u \\
= & -\beta \sigma_{r} \int_{0}^{T}\left(\int_{0}^{u} \mathrm{e}^{\beta s} \mathrm{~d} Z_{s}^{r}\right)(T-u) \mathrm{e}^{-\beta u} \mathrm{~d} u=-\beta \sigma_{r} \int_{0}^{T}\left(\int_{0}^{u} \mathrm{e}^{\beta s} \mathrm{~d} Z_{s}^{r}\right) \mathrm{d}\left(\int_{0}^{u}(T-v) \mathrm{e}^{-\beta v} \mathrm{~d} v\right) \\
= & -\beta \sigma_{r}\left[\left(\int_{0}^{T} \mathrm{e}^{\beta u} \mathrm{~d} Z_{u}^{r}\right)\left(\int_{0}^{T}(T-v) \mathrm{e}^{-\beta v} \mathrm{~d} v\right)-\int_{0}^{T}\left(\int_{0}^{u}(T-v) \mathrm{e}^{-\beta v} \mathrm{~d} v\right) \mathrm{e}^{\beta u} \mathrm{~d} Z_{u}^{r}\right] \\
= & -\beta \sigma_{r}\left[\int_{0}^{T} \mathrm{e}^{\beta u}\left(\int_{0}^{u}(T-v) \mathrm{e}^{-\beta v} \mathrm{~d} v\right) \mathrm{d} Z_{u}^{r}\right]=-\frac{\sigma_{r}}{\beta}\left[\int_{0}^{T}\left(\mathrm{e}^{-\beta(T-u)}-1\right) \mathrm{d} Z_{u}^{r}\right]-\sigma_{r} \int_{0}^{T}(T-u) \mathrm{d} Z_{u}^{r} .
\end{aligned}
$$


The terminal payoff of a European option on the underlying stock $S_{t}$ with strike price $K$ is

$$
\max \left(S_{T}-K, 0\right)
$$

This means the holder will exercise his right only $S_{T}>K$ and then his gain is $S_{T}-K$. Otherwise, if $S_{T} \leq K$ then the holder will buy the underlying asset from the market and the value of the option is zero.

We would like to find a formula for pricing a European call option with strike price $K$ and maturity $T$ based on the model (3.25). Consider a continuous-time economy where interest rates are stochastic and the price of the European call option at time $t$ under the $T$-forward measure $Q^{T}$ is

$$
\begin{aligned}
& C\left(t, S_{t}, r_{t}, v_{t} ; T, K\right)=P(t, T) E_{Q^{T}}\left(\max \left(S_{T}-K, 0\right) \mid S_{t}, r_{t}, v_{t}\right) \\
& =P(t, T) \int_{0}^{\infty} \max \left(S_{T}-K, 0\right) p_{Q^{T}}\left(S_{T} \mid S_{t}, r_{t}, v_{t}\right) \mathrm{d} S_{T}
\end{aligned}
$$

where $E_{Q^{T}}$ is the expectation with respect to the $T$-forward probability measure, $p_{Q^{T}}$ is the corresponding conditional density given $\left(S_{t}, r_{t}, v_{t}\right)$ and $P$ is a zero coupon bond which is defined in Lemma 1.

With a change in variable $X_{t}=\ln S_{t}$,

$$
\begin{aligned}
& C\left(t, S_{t}, r_{t}, v_{t} ; T, K\right) \\
= & P(t, T) \int_{-\infty}^{\infty} \max \left(\mathrm{e}^{X_{T}}-K, 0\right) p_{Q^{T}}\left(X_{T} \mid X_{t}, r_{t}, v_{t},\right) \mathrm{d} X_{T} \\
= & P(t, T) \int_{\ln K}^{\infty}\left(\mathrm{e}^{X_{T}}-K\right) 1_{X_{T} \geq \ln K} p_{Q^{T}}\left(X_{T} \mid X_{t}, r_{t}, v_{t}\right) \mathrm{d} X_{T} \\
= & P(t, T) \int_{\ln K}^{\infty} \mathrm{e}^{X_{T}} p_{Q^{T}}\left(X_{T} \mid X_{t}, r_{t}, v_{t}\right) \mathrm{d} X_{T} \\
& -K P(t, T) \int_{\ln K}^{\infty} p_{Q^{T}}\left(X_{T} \mid X_{t}, r_{t}, v_{t}\right) \mathrm{d} X_{T} \\
= & \mathrm{e}^{X_{t}}\left(\frac{1}{E_{Q^{T}}\left(\mathrm{e}^{X_{T}} \mid S_{t}, r_{t}, v_{t}\right)} \int_{\ln K}^{\infty} \mathrm{e}^{X_{T}} p_{Q^{T}}\left(X_{T} \mid X_{t}, r_{t}, v_{t}\right) v X_{T}\right) \\
& -K P(t, T) \int_{\ln K}^{\infty} p_{Q^{T}}\left(X_{T} \mid X_{t}, r_{t}, v_{t}\right) \mathrm{d} X_{T} \\
= & \mathrm{e}^{X_{t}}\left(\int_{\ln K}^{\infty} e^{X_{T}} \frac{p_{Q^{T}}\left(X_{T} \mid X_{t}, r_{t}, v_{t}\right)}{E_{Q^{T}}\left(\mathrm{e}^{X_{T}} \mid S_{t}, r_{t}, v_{t}\right)} \mathrm{d} X_{T}\right)
\end{aligned}
$$

$$
-K P(t, T) \int_{\ln K}^{\infty} p_{Q^{T}}\left(X_{T} \mid X_{t}, r_{t}, v_{t}\right) \mathrm{d} X_{T}
$$

With the first integrand in (4.2) being positive and integrating up to one. The first integrand therefore defines a new probability measure that we denote by $q_{Q^{T}}$ below

$$
\begin{aligned}
& C\left(t, S_{t}, r_{t}, v_{t} ; T, K\right) \\
= & \mathrm{e}^{X_{t}} \int_{\ln K}^{\infty} q_{Q^{T}}\left(X_{T} \mid X_{t}, r_{t}, v_{t}\right) \mathrm{d} X_{T} \\
& -K P(t, T) \int_{\ln K}^{\infty} p_{Q^{T}}\left(X_{T} \mid X_{t}, r_{t}, v_{t}\right) \mathrm{d} X_{T} \\
= & \mathrm{e}^{X_{t}} \mathrm{P}_{1}\left(t, X_{t}, r_{t}, v_{t} ; T, K\right)-K P(t, T) \mathrm{P}_{2}\left(t, X_{t}, r_{t}, v_{t} ; T, K\right) \\
= & \mathrm{e}^{X_{t}} \operatorname{Pr}\left(X_{T}>\ln K \mid X_{t}, r_{t}, v_{t}\right) \\
& -K P(t, T) \operatorname{Pr}\left(X_{T}>\ln K \mid X_{t}, r_{t}, v_{t}\right)
\end{aligned}
$$

where those probabilities in (4.3) are calculated under the probability measure $Q^{T}$.

The European call option for log asset price $X_{t}=\ln S_{t}$ will be denoted by

$$
\begin{aligned}
\hat{C}\left(t, X_{t}, r_{t}, v_{t} ; T, \kappa\right)= & \mathrm{e}^{X_{t}} \tilde{\mathrm{P}}_{1}\left(t, X_{t}, r_{t}, v_{t} ; T, \kappa\right) \\
& -\mathrm{e}^{\kappa} P(t, T) \tilde{\mathrm{P}}_{2}\left(t, X_{t}, r_{t}, v_{t} ; T, \kappa\right)
\end{aligned}
$$

where $\kappa=\ln K$ and

$$
\tilde{\mathrm{P}}_{j}\left(t, X_{t}, r_{t}, v_{t} ; T, \kappa\right):=\mathrm{P}_{j}\left(t, X_{t}, r_{t}, v_{t} ; T, K\right), j=1,2 .
$$

Note that we do not have a closed form solution for these probabilities. However, these probabilities are related to characteristic functions which have closed form solutions as will be seen in Lemma 4. The following lemma shows the relationship between $\tilde{P}_{1}$ and $\tilde{P}_{2}$ in the option value of (4.4).

Lemma 3 The functions $\tilde{P}_{1}$ and $\tilde{P}_{2}$ in the option values of (4.4) satisfy the PIDEs (4.5):

and subject to the boundary condition at expiration $t=T$

$$
\tilde{P}_{1}(T, x, r, v ; T, \kappa)=1_{x>\kappa} .
$$

Moreover, $\tilde{P}_{2}$ satisfies the Equation (4.7)

$$
\begin{aligned}
0= & \frac{\partial \tilde{P}_{1}}{\partial t}+A\left[\tilde{P}_{1}\right]+\left(\rho_{v} \sigma v \sigma_{v}\right) \frac{\partial \tilde{P}_{1}}{\partial v}+v \int_{-\infty}^{\infty}\left[\left(\mathrm{e}^{y}-1\right)\left(\tilde{P}_{1}(t, x+y, r, v ; T, \kappa)-\tilde{P}_{1}(x, t, r, v ; T, \kappa)\right)\right] k(y) \mathrm{d} y \\
0= & \frac{\partial \tilde{P}_{2}}{\partial t}+A\left[\tilde{P}_{2}\right]-\sigma^{2} v \frac{\partial v}{\partial x}+\frac{\sigma^{2} v}{2} \frac{\partial^{2} \tilde{P}_{2}}{\partial x^{2}}+b(t, T) \sigma_{r}^{2} \frac{\partial \tilde{P}_{2}}{\partial r} \\
& +\tilde{P}_{2}\left(\frac{\partial a(t, T)}{\partial t}+r\left(\frac{\partial b(t, T)}{\partial t}-1\right)+\frac{\sigma_{r}^{2}}{2} b^{2}(t, T)\right)+\tilde{P}_{2}\left(\alpha-\beta r-\frac{\sigma_{r}^{2}}{\beta}\left(1-\mathrm{e}^{-\beta(T-t)}\right)\right) b(t, T) \\
& +v \int_{-\infty}^{\infty}\left(\widetilde{P}_{2}(t, x+y, r, v ; T, \kappa)-\tilde{P}_{2}(t, x, r, v ; T, \kappa)-\frac{\partial \tilde{P}_{2}}{\partial x}\left(\mathrm{e}^{y}-1\right)\right) k(y) \mathrm{d} y
\end{aligned}
$$


and subject to the boundary condition at expiration $t=T$

$$
\tilde{P}_{2}(T, x, r, v ; T, \kappa)=1_{x>\kappa}
$$

where for $i=1,2$

$$
\begin{aligned}
A\left[\tilde{P}_{i}\right]= & \left(r+\frac{1}{2} \sigma^{2} v\right) \frac{\partial \tilde{P}_{i 1}}{\partial x}+\left(\alpha-\beta r-\frac{\sigma_{r}^{2}}{\beta}\left(1-\mathrm{e}^{-\beta(T-t)}\right)\right) \frac{\partial \tilde{P}_{i}}{\partial r} \\
& +\gamma(1-v) \frac{\partial \tilde{P}_{i}}{\partial v}+\frac{\sigma_{v}^{2} v}{2} \frac{\partial^{2} \tilde{P}_{i}}{\partial v^{2}}+\frac{\sigma^{2} v}{2} \frac{\partial^{2} \tilde{P}_{i}}{\partial x^{2}}+\frac{\sigma_{r}^{2}}{2} \frac{\partial^{2} \tilde{P}_{i}}{\partial r^{2}}+\left(\rho_{v} \sigma v \sigma_{v}\right) \frac{\partial \tilde{P}_{i}}{\partial v \partial x} \\
& +v \int_{-\infty}^{\infty}\left[\tilde{P}_{i}(t, x+y, r, v ; T, \kappa)-\tilde{P}_{i}(x, t, r, v ; T, \kappa)-\left(\frac{\partial \tilde{P}_{i}}{\partial x}\right)\left(\mathrm{e}^{y}-1\right)\right] k(y) \mathrm{d} y
\end{aligned}
$$

Note that $1_{x>\kappa}=1$ if $x>\kappa$ and otherwise $1_{x>\kappa}=0$.

Proof. See Appendix A.

\section{The Closed-Form Solution for European Call Options}

For $j=1,2$ the characteristic function for $\tilde{P}_{j}(t, x, r, v ; T, \kappa)$, with respect to the variable $\kappa$, are defined by

$$
f_{j}(t, x, r, v ; T, u):=-\int_{-\infty}^{\infty} \mathrm{e}^{\mathrm{iu \kappa}} \mathrm{d} \tilde{P}_{j}(t, x, r, v ; T, \kappa),
$$

with a minus sign to account for the negativity of the measure $d \tilde{P}_{j}$. Note that $f_{j}$ also satisfies similar PIDEs

$$
\frac{\partial f_{j}}{\partial t}+A_{j}\left[f_{j}\right](t, x, r, v ; T, \kappa)=0,
$$

with the respective boundary conditions

$$
\begin{aligned}
f_{j}(T, x, r, v ; T, u) & =-\int_{-\infty}^{\infty} \mathrm{e}^{i u \kappa} \mathrm{d} \tilde{P}_{j}(t, x, r, v ; T, \kappa) \\
& =-\int_{-\infty}^{\infty} \mathrm{e}^{i u \kappa}(-\delta(\kappa-x)) \mathrm{d} \kappa=\mathrm{e}^{i u x} .
\end{aligned}
$$

Since $\mathrm{d} \tilde{P}_{j}(t, x, r, v ; T, \kappa)=d 1_{x>\kappa}=-\delta(\kappa-x) \mathrm{d} \kappa$

The following lemma shows how to calculate the characteristic functions for $\tilde{P}_{1}$ and $\tilde{P}_{2}$ as they appeared in Lemma 3.

Lemma 4 The functions $\tilde{P}_{1}$ and $\tilde{P}_{2}$ can be calculated by the inverse Fourier transformations of the characteristic function, i.e.

$$
\tilde{P}_{j}(t, x, r, v ; T, \kappa)=\frac{1}{2}+\frac{1}{\pi} \int_{0+}^{\infty} \operatorname{Re}\left[\frac{\mathrm{e}^{i u \kappa} f_{j}(t, x, r, v ; T, u)}{i u}\right] \mathrm{d} u,
$$

for $j=1,2$, with $\mathrm{Re}[$.$] denoting the real component of a$ complex number.

By letting $\tau=T-t$, the characteristic function $f_{j}$ is given by $f_{j}(t, x, r, v ; t+\tau, u)=\exp \left(\mathrm{iux}+B_{j}(\tau)+r C_{j}(\tau)+v E_{j}(\tau)\right)$, where

$$
\begin{gathered}
\tilde{b}_{j 1}=b_{j 2}+\nabla_{j}, \quad \tilde{b}_{j 2}=b_{j 2}-\nabla_{j}, \quad b_{j 1}=\frac{\sigma_{v}^{2}}{2}, \\
b_{12}=\rho_{v} \sigma \sigma_{v}(1+i u)-\gamma, b_{22}=\rho_{v} \sigma \sigma_{v} i u-\gamma, \\
b_{10}=-\left(\frac{1}{2} \sigma^{2}\left(i u-u^{2}\right)+\int_{-\infty}^{\infty}\left(\mathrm{e}^{i u x+y}-i u\left(\mathrm{e}^{y}-1\right)\right) k(y) \mathrm{d} y\right) \\
b_{20}=-\left(\frac{1}{2} \sigma^{2}\left(u^{2}+i u\right)-\int_{-\infty}^{\infty}\left(\mathrm{e}^{i u x}-i u\left(\mathrm{e}^{y}-1\right)\right) k(y) \mathrm{d} y\right) \\
B_{1}(\tau)=\frac{-\tau \gamma \tilde{b}_{12}}{2 b_{11}}+\frac{\gamma\left(\tilde{b}_{11}+\tilde{b}_{12}\right)}{2 b_{11} \nabla_{1}} \ln \left(\frac{\tilde{b}_{11}+\mathrm{e}^{\tau \nabla_{1}} \tilde{b}_{12}}{\tilde{b}_{11}+\tilde{b}_{12}}\right) \\
+\frac{i u \sigma_{r}^{2}}{2 \beta^{3}}\left(\mathrm{e}^{-\beta \tau}-1\right)^{2}+\left(2 i u\left(\alpha \beta-\sigma_{r}^{2}\right)-\sigma_{r}^{2} u^{2}\right) \frac{\tau}{\beta^{2}} \\
+\frac{\sigma_{r}^{2} u}{4 \beta^{3}}\left(-4 \mathrm{e}^{-\beta \tau}+\mathrm{e}^{-2 \beta \tau}-3\right) \\
C_{j}(\tau)=\frac{i u}{\beta}\left(1-\mathrm{e}^{-\beta \tau}\right), \nabla_{j}=\sqrt{b_{j 2}{ }^{2}-4 b_{j 0} b_{j 1}} \\
E_{j}(\tau)=\frac{\tilde{b}_{j 1} \tilde{b}_{j 2}\left(\mathrm{e}^{\left.\tau \sqrt{b_{j 2}{ }^{2}-4 b_{j 0} b_{j 1}}-1\right)}\right.}{2 b_{j 1}\left(\tilde{b}_{j 1}+\tilde{b}_{j 2} \mathrm{e}^{\tau \sqrt{b_{j 2}{ }^{2}-4 b_{j 0} b_{j 1}}}\right)} .
\end{gathered}
$$

$$
\begin{aligned}
B_{2}(\tau)= & \frac{-\tau \gamma \tilde{b}_{22}}{2 b_{21}}+\frac{\gamma\left(\tilde{b}_{22}+\tilde{b}_{21}\right)}{2 b_{21} \nabla_{2}} \ln \left(\frac{\tilde{b}_{21}+e^{\tau \nabla} 2 \tilde{b}_{22}}{\tilde{b}_{21}+\tilde{b}_{22}}\right) \\
& \left.+\left(\frac{\sigma_{r}^{2}}{\beta^{3}}\left(u^{2}+4 i u-2\right)-\frac{i u \alpha}{\beta^{2}}\right)\left(1-\mathrm{e}^{-\beta \tau}\right)\right) \\
& +\frac{\sigma_{r}^{2}}{4 \beta^{3}}\left(u^{2}+4 i u-2\right)\left(\mathrm{e}^{-2 \beta \tau}-1\right) \\
& +\left(\frac{\alpha}{\beta^{2}}(i u \beta-\beta+1)-\frac{\sigma_{r}^{2}}{2 \beta^{2}}\left(u^{2}+4 i u-2\right)\right) \tau
\end{aligned}
$$

Proof. See Appendix B.

In summary, we have just proved the following main theorem. 
Theorem 5 The value of a European call option of $S D E(3.25)$ is

$$
\begin{aligned}
& C\left(t, S_{t}, r_{t}, v_{t} ; T, K\right)= \\
& S_{t} \tilde{P}_{1}\left(t, X_{t}, r_{t}, v_{t} ; T, \kappa\right)-K P(t, T) \tilde{P}_{2}\left(t, X_{t}, r_{t}, v_{t} ; T, \kappa\right)
\end{aligned}
$$

where $\tilde{P}_{1}$ and $\tilde{P}_{2}$ are given in Lemma 4 and $P(t, T)$ is given in Lemma 1.

\section{Acknowledgements}

This research is (partially) supported by The Center of Excellent in Mathematics, the commission on Higher Education (CHE).

Address: 272 Rama VI Road, Ratchathewi District, Bangkok, Thailand.

\section{References}

[1] P. Carr and L. Wu, "Time Change Levy Processes and Option Pricing," Journal of Financial Economics, Vol. 17, No. 1, 2004, pp. 113-141. doi:10.1016/S0304-405X(03)00171-5

[2] R. C. Merton, "Option Pricing when Underlying Stock Returns are Discontinuous," Journal of Financial Economics, Vol. 3, No. 1-2, 1976, pp. 125-144. doi:10.1016/0304-405X(76)90022-2
[3] D. Bates, "Jump and Stochastic Volatility: Exchange Rate Processes Implicit in Deutche Mark in Option," Review of Financial Studies, Vol. 9, No. 1, 1996, pp. 69-107. doi:10.1093/rfs/9.1.69

[4] G. Yan and F. B. Hanson, "Option Pricing for Stochastic Volatility Jump Diffusion Model with Log Uniform Jump Amplitudes," Proceeding American Control Conference, Minneapolis, 14-16 June 2006, pp. 2989-2994.

[5] Y. J. Kim, "Option Pricing under Stochastic Interest rates: An Empirical Investigation," Asia Pacific Financial Markets, Vol. 9, No. 1, 2001, pp. 23-44. doi:10.1023/A:1021155301176

[6] D. Brigo and F. Mercuiro, "Interest Rate Models: Theory and Practice," 2nd Edition, Springer, Berlin, 2001.

[7] R. Brummelhuis, "Mathematical Method for Financial Engineering," University of London, 2009.

http://www.ems.bbk.ac.uk/for_students/msc./math_metho ds/lecture1.pdf

[8] P.E. Plotter, "Stochastic Integration and Differential Equation," Stochastic Modeling and Applied Probability, Vol. 21, 2nd Edition, Springer, Berlin, 2005.

[9] N. Privault, "An Elementary Introduction to Stochastic Interest Rate Modeling," Advance Series on Statistical Science \& Applied Probability, Vol. 2, World Scientific, Singapore, 2008.

[10] M. G. Kendall, A. Stuat and J. K. Ord, "Advance Theory of Statistics Vol. 1," Halsted Press, New York, 1987. 
Appendix A: Proof of Lemma 3

By Ito's lemma, $\hat{C}(t, x, r, v)$ follows the partial integro-differential equation (PIDE)

where

$$
\frac{\partial \hat{C}}{\partial t}+L_{t}^{D} \hat{C}+L_{t}^{J} \hat{C}=0
$$

$$
\begin{aligned}
L_{t}^{D} \hat{C}= & \left(r-\frac{1}{2} \sigma^{2} v\right) \frac{\partial \hat{C}}{\partial x}+\left(\alpha-\beta r-\frac{\sigma_{r}^{2}}{\beta}\left(1-\mathrm{e}^{-\beta(T-t)}\right)\right) \frac{\partial \hat{C}}{\partial r} \\
& +\gamma(1-v) \frac{\partial \hat{C}}{\partial v}+\frac{\sigma_{v}^{2} v}{2} \frac{\partial^{2} \hat{C}}{\partial v^{2}}+\frac{\sigma^{2} v}{2} \frac{\partial^{2} \hat{C}}{\partial x^{2}}+\frac{\sigma_{r}^{2}}{2} \frac{\partial^{2} \hat{C}}{\partial r^{2}} \\
& +\left(\rho_{v} \sigma v \sigma_{v}\right) \frac{\partial^{2} \hat{C}}{\partial x \partial v}-r \hat{C}
\end{aligned}
$$

and

$$
\begin{aligned}
& L_{t}^{J} \hat{C} \\
= & v \int_{-\infty}^{\infty}\left(\hat{C}(t, x+y, r, v)-\hat{C}(t, x, r, v)-\frac{\partial \hat{C}}{\partial x}\left(\mathrm{e}^{y}-1\right)\right) k(y) \mathrm{d} y
\end{aligned}
$$

where $k(y)$ is the Lévy density.

We plan to substitute (4.4) into (A.1). Firstly, we compute

$$
\frac{\partial \hat{C}}{\partial t}=\mathrm{e}^{x} \frac{\partial \tilde{P}_{1}}{\partial t}-\mathrm{e}^{\kappa} P(t, T)\left[\frac{\partial \tilde{P}_{2}}{\partial t}+\tilde{P}_{2} \frac{\partial}{\partial t}(a(t, T)+b(t, T) r)\right],
$$$$
\frac{\partial \hat{C}}{\partial x}=\mathrm{e}^{x}\left(\frac{\partial \tilde{P}_{1}}{\partial x}+\tilde{P}_{1}\right)-\mathrm{e}^{\kappa} P(t, T) \frac{\partial \tilde{P}_{2}}{\partial x},
$$$$
\frac{\partial \hat{C}}{\partial v}=\mathrm{e}^{x} \frac{\partial \tilde{P}_{1}}{\partial v}-\mathrm{e}^{\kappa} P(t, T) \frac{\partial \tilde{P}_{2}}{\partial v},
$$$$
\frac{\partial \hat{C}}{\partial r}=\mathrm{e}^{x} \frac{\partial \tilde{P}_{1}}{\partial r}-\mathrm{e}^{\kappa} P(t, T)\left(\frac{\partial \tilde{P}_{2}}{\partial r}+\tilde{P}_{2} b(t, T)\right),
$$$$
\frac{\partial^{2} \hat{C}}{\partial x^{2}}=\mathrm{e}^{x}\left(\frac{\partial^{2} \tilde{P}_{1}}{\partial x^{2}}+2 \frac{\partial \tilde{P}_{1}}{\partial x}+\tilde{P}_{1}\right)-\mathrm{e}^{\kappa} P(t, T) \frac{\partial^{2} \tilde{P}_{2}}{\partial x^{2}},
$$$$
\frac{\partial^{2} \hat{C}}{\partial v^{2}}=\mathrm{e}^{x} \frac{\partial^{2} \tilde{P}_{1}}{\partial v^{2}}-\mathrm{e}^{\kappa} P(t, T) \frac{\partial^{2} \tilde{P}_{2}}{\partial v^{2}},
$$$$
\frac{\partial^{2} \hat{C}}{\partial v \partial x}=\mathrm{e}^{x}\left(\frac{\partial \tilde{P}_{1}}{\partial v \partial x}+\frac{\partial \tilde{P}_{1}}{\partial v}\right)-\mathrm{e}^{\kappa} P(t, T) \frac{\partial \tilde{P}_{2}}{\partial v \partial x}, \frac{\partial^{2} \hat{C}}{\partial r^{2}}=\mathrm{e}^{x} \frac{\partial^{2} \tilde{P}_{1}}{\partial r^{2}}
$$

$$
-\mathrm{e}^{\kappa} P(t, T)\left(\frac{\partial^{2} \tilde{P}_{2}}{\partial r^{2}}+2 b(t, T) \frac{\partial \tilde{P}_{2}}{\partial r}+\tilde{P}_{2} b^{2}(t, T)\right), .
$$

$$
\frac{\partial^{2} \hat{C}}{\partial x \partial r}=\mathrm{e}^{x}\left(\frac{\partial^{2} \tilde{P}_{1}}{\partial x \partial r}+\frac{\partial \tilde{P}_{1}}{\partial r}\right)-\mathrm{e}^{\kappa} P(t, T)\left(\frac{\partial^{2} \tilde{P}_{2}}{\partial x \partial r}+b(t, T) \frac{\partial \tilde{P}_{2}}{\partial x}\right),
$$

$$
\begin{aligned}
& \hat{C}(t, x+y, r, v, ; T, \kappa)-\hat{C}(t, x, r, v, ; T, \kappa) \\
= & \mathrm{e}^{x}\left[\left(\mathrm{e}^{y}-1\right) \tilde{P}_{1}(t, x+y, r, v ; T, \kappa)+\left(\tilde{P}_{1}(t, x+y, r, v ; T, \kappa)-\tilde{P}_{1}(x, t, r, v ; T, \kappa)\right)\right] \\
& -\mathrm{e}^{\kappa} P(t, T)\left[\tilde{P}_{2}(t, x+y, r, v ; T, \kappa)-\tilde{P}_{2}(t, x, r, v ; T, \kappa)\right] .
\end{aligned}
$$

Substitute all terms above into (A.1) and separate it by assumed independent terms of $\tilde{P}_{1}$ and $\tilde{P}_{2}$. This gives

two PIDEs for the $T$-forward probability for

$$
\tilde{P}_{i}(t, x, r, v ; T, \kappa), j=1,2 \text { : }
$$

$$
\begin{aligned}
& \frac{\partial \tilde{P}_{1}}{\partial t}+\left(r+\frac{1}{2} \sigma^{2} v\right) \frac{\partial \tilde{P}_{1}}{\partial x}+\left(\alpha-\beta r-\frac{\sigma_{r}^{2}}{\beta}\left(1-\mathrm{e}^{-\beta(T-t)}\right)\right) \frac{\partial \tilde{P}_{1}}{\partial r} \\
& +\left(\rho_{v} \sigma v \sigma_{v}\right) \frac{\partial \tilde{P}_{1}}{\partial v \partial x}+\frac{\sigma_{v}^{2} v}{2} \frac{\partial^{2} \tilde{P}_{1}}{\partial v^{2}}+\frac{\sigma^{2} v}{2} \frac{\partial^{2} \tilde{P}_{1}}{\partial x^{2}}+\frac{\sigma_{r}^{2}}{2} \frac{\partial^{2} \tilde{P}_{1}}{\partial r^{2}}+\left(\gamma(1-v)+\rho_{v} \sigma v \sigma_{v}\right) \frac{\partial \tilde{P}_{1}}{\partial v} \\
& +v \int_{-\infty}^{\infty}\left[\tilde{P}_{1}(t, x+y, r, v ; T, \kappa)-\tilde{P}_{1}(x, t, r, v ; T, \kappa)-\left(\frac{\partial \tilde{P}_{1}}{\partial x}\right)\left(\mathrm{e}^{y}-1\right)\right] k(y) \mathrm{d} y . \\
& +v \int_{-\infty}^{\infty}\left[\left(\mathrm{e}^{y}-1\right)\left(\tilde{P}_{1}(t, x+y, r, v ; T, \kappa)-\tilde{P}_{1}(x, t, r, v ; T, \kappa)\right)\right] k(y) \mathrm{d} y .=0
\end{aligned}
$$

and subject to the boundary condition at the expiration time $t=T$ according to (4.6).

$$
\begin{aligned}
0 & =\frac{\partial \tilde{P}_{1}}{\partial t}+A\left[\tilde{P}_{1}\right]+\left(\rho_{v} \sigma v \sigma_{v}\right) \frac{\partial \tilde{P}_{1}}{\partial v}+v \int_{-\infty}^{\infty}\left[\left(\mathrm{e}^{y}-1\right)\left(\tilde{P}_{1}(t, x+y, r, v ; T, \kappa)-\tilde{P}_{1}(x, t, r, v ; T, \kappa)\right)\right] k(y) \mathrm{d} y . \\
& :=\frac{\partial \tilde{P}_{1}}{\partial t}+A_{1}\left[\tilde{P}_{1}\right] .
\end{aligned}
$$


For $\tilde{P}_{2}(t, x, r, v ; T, \kappa)$ :

$$
\begin{aligned}
0= & \frac{\partial \tilde{P}_{2}}{\partial t}+\left(r-\frac{1}{2} \sigma^{2} v\right) \frac{\partial \tilde{P}_{2}}{\partial x}+\gamma(1-v) \frac{\partial \tilde{P}_{2}}{\partial v}+\left(\rho_{v} \sigma v \sigma_{v}\right) \frac{\partial \tilde{P}_{2}}{\partial v \partial x}+\frac{\sigma^{2} v}{2} \frac{\partial^{2} \tilde{P}_{2}}{\partial x^{2}}+\frac{\sigma_{v}^{2} v}{2} \frac{\partial^{2} \tilde{P}_{2}}{\partial v^{2}}+\frac{\sigma_{r}^{2}}{2} \frac{\partial^{2} \tilde{P}_{2}}{\partial r^{2}}+\left(\frac{\sigma_{r}^{2}}{2} b^{2}(t, T)-r\right) \tilde{P}_{2} \\
& +\left(\alpha-\beta r-\frac{\sigma_{r}^{2}}{\beta}\left(1-e^{-\beta(T-t)}\right)+2 b(t, T) \frac{\sigma_{r}^{2}}{2}\right)\left(\frac{\partial \tilde{P}_{2}}{\partial r}\right)+\tilde{P}_{2}\left(\frac{\partial a(t, T)}{\partial t}+r \frac{\partial b(t, T)}{\partial t}+\left(\alpha-\beta r-\frac{\sigma_{r}^{2}}{\beta}\left(1-\mathrm{e}^{-\beta(T-t)}\right)\right) b(t, T)\right) \\
& +v \int_{-\infty}^{\infty}\left(\tilde{P}_{2}(t, x+y, r, v ; T, \kappa)-\tilde{P}_{2}(t, x, r, v ; T, \kappa)-\frac{\partial \tilde{P}_{2}}{\partial x}\left(\mathrm{e}^{y}-1\right)\right) k(y) \mathrm{d} y .
\end{aligned}
$$

and subject to the boundary condition at expiration time $t$ $=T$ according to (4.8).

$$
\begin{aligned}
0= & \frac{\partial \tilde{P}_{2}}{\partial t}+A\left[\tilde{P}_{2}\right]-\sigma^{2} v \frac{\partial \tilde{P}_{2}}{\partial x}+\frac{\sigma^{2} v}{2} \frac{\partial^{2} \tilde{P}_{2}}{\partial x^{2}}+b(t, T) \sigma_{r}^{2} \frac{\partial \tilde{P}_{2}}{\partial r}+\tilde{P}_{2}\left(\frac{\partial a(t, T)}{\partial t}+r\left(\frac{\partial b(t, T)}{\partial t}-1\right)+\frac{\sigma_{r}^{2}}{2} b^{2}(t, T)\right) \\
& +\tilde{P}_{2}\left(\alpha-\beta r-\frac{\sigma_{r}^{2}}{\beta}\left(1-\mathrm{e}^{-\beta(T-t)}\right)\right) b(t, T):=\frac{\partial \tilde{P}_{2}}{\partial t}+A_{2}\left[\tilde{P}_{2}\right]
\end{aligned}
$$

Again, by using the notation (4.9), then (A.4) becomes

The proof is now completed. 
Appendix B: Proof of Lemma 4

To solve the characteristic function explicitly, letting $\tau=T-t$ be the time-to-go, we conjecture that the function $f_{1}$ is given by

$$
\begin{aligned}
& f_{1}(t, x, r, v ; t+\tau, u) \\
= & \exp \left(\operatorname{iux}+B_{1}(\tau)+r C_{1}(\tau)+v E_{1}(\tau)\right),
\end{aligned}
$$

and the boundary condition $B_{1}(0)=C_{1}(0)=E_{1}(0)=0$. This conjecture exploits the linearity of the coefficient in PIDEs (5.2).

Note that the characteristic function of $f_{1}$ always exists. In order to substitute (B.1) into (5.2), firstly, we compute

$$
\begin{aligned}
& \frac{\partial f_{1}}{\partial t}=-\left(B_{1}^{\prime}(\tau)+r C_{1}^{\prime}(\tau)+v E_{1}^{\prime}(\tau)\right) f_{1}, \quad \frac{\partial f_{1}}{\partial x}=i u f_{1}, \\
& 0=r\left[-C_{1}^{\prime}(\tau)+i u-\beta C_{1}(\tau)\right] \\
& +v\left[-E_{1}^{\prime}(\tau)+\left(\rho_{v} \sigma \sigma_{v}(1+i u)-\gamma\right) E_{1}(\tau)+\frac{\sigma_{v}^{2}}{2} E_{1}^{2}(\tau)+\frac{\sigma^{2}}{2}\left(i u-u^{2}\right)+\int_{-\infty}^{\infty}\left[\mathrm{e}^{i u x}-i u\left(\mathrm{e}^{y}-1\right)+\left(\mathrm{e}^{y}-1\right) \mathrm{e}^{i u x}\right] k(y) \mathrm{d} y\right] \\
& +\left[-B_{1}^{\prime}(\tau)+\left(\alpha-\frac{\sigma_{r}^{2}}{\beta}\left(1-\mathrm{e}^{-\beta(T-t)}\right)\right) C_{1}(\tau)+\frac{\sigma_{r}^{2}}{2} C_{1}^{2}(\tau)+\gamma E_{1}(\tau)\right]
\end{aligned}
$$

$$
\begin{aligned}
& \frac{\partial f_{1}}{\partial r}=C_{1}(\tau) f_{1}, \quad \frac{\partial f_{1}}{\partial v}=E_{1}(\tau) f_{1}, \quad \frac{\partial^{2} f_{1}}{\partial x^{2}}=-u^{2} f_{1}, \\
& \frac{\partial^{2} f_{1}}{\partial v^{2}}=E_{1}^{2}(\tau) f_{1}, \frac{\partial^{2} f_{1}}{\partial r^{2}}=C_{1}^{2}(\tau) f_{1}, \\
& \frac{\partial^{2} f_{1}}{\partial x \partial r}=i u C_{1}(\tau) f_{1}, \quad \frac{\partial^{2} f_{1}}{\partial v \partial x}=i u E_{1}(\tau) f_{1}, \\
& \mathrm{e}^{i u x} f_{1}(t, x, r, v ; t+\tau, u) \\
& =f_{1}(t, x+y, r, v ; t+\tau, u)-f_{1}(t, x, r, v ; t+\tau, u)
\end{aligned}
$$

Substituting all the above terms into (5.2), after can-
By separating the order, $r, v$ and ordering the remaining terms, we can reduce it to three ordinary differential equations (ODEs) as follows:

$$
\begin{gathered}
C_{1}^{\prime}(\tau)=-\beta C_{1}(\tau)+i u, \\
E_{1}^{\prime}(\tau)=\frac{\sigma_{v}^{2}}{2} E_{1}^{2}(\tau)+\left(\rho_{v} \sigma \sigma_{v}(1+i u)-\gamma\right) E_{1}(\tau) \\
+\frac{\sigma^{2}}{2}\left(i u-u^{2}\right)+\int_{-\infty}^{\infty}\left(\mathrm{e}^{i u x+y}-i u\left(\mathrm{e}^{y}-1\right)\right) k(y) \mathrm{d} y,(\mathrm{~B} .3) \\
B_{1}^{\prime}(\tau)=\left[\alpha-\frac{\sigma_{r}^{2}}{\beta}\left(1-\mathrm{e}^{-\beta(T-t)}\right)\right] C_{1}(\tau)+\gamma E_{1}(\tau)+\frac{\sigma_{r}^{2}}{2} C_{1}^{2}(\tau) .
\end{gathered}
$$

It is clear from (B.2) and $C(0)=0$ that

$$
C_{1}(\tau)=\frac{i u}{\beta} \times\left(1-\mathrm{e}^{-\beta \tau}\right),
$$

Let

$$
\begin{gathered}
b_{1}=\frac{\sigma_{v}^{2}}{2}, \\
b_{2}=\rho_{v} \sigma \sigma_{v}(1+i u)-\gamma,
\end{gathered}
$$

$b_{0}=-\left(\frac{1}{2} \sigma^{2}\left(i u-u^{2}\right)+\int_{-\infty}^{\infty}\left(\mathrm{e}^{i u x+y}-i u\left(\mathrm{e}^{y}-1\right)\right) k(y) \mathrm{d} y\right)$

and substitute all term above into (B.3). we get

$$
E_{1}^{\prime}(\tau)=b_{1}\left(E_{1}(\tau)-\frac{-b_{2}+\sqrt{b_{2}^{2}-4 b_{0} b_{1}}}{2 b_{1}}\right) \times\left(E_{1}(\tau)-\frac{-b_{2}-\sqrt{b_{2}^{2}-4 b_{0} b_{1}}}{2 b_{1}}\right)
$$

By method of variable separation, we have

$$
\frac{\mathrm{d} E_{1}(\tau)}{\left(E_{1}(\tau)-\frac{-b_{2}+\sqrt{b_{2}^{2}-4 b_{0} b_{1}}}{2 b_{1}}\right)\left(E_{1}(\tau)-\frac{-b_{2}-\sqrt{b_{2}^{2}-4 b_{0} b_{1}}}{2 b_{1}}\right)}=b_{1} \mathrm{~d} \tau
$$

Using partial fraction on the left hand side, we get 
$\left(\frac{1}{\left(E_{1}(\tau)-\frac{-b_{2}+\nabla}{2 b_{1}}\right)}-\frac{1}{\left(E_{1}(\tau)-\frac{-b_{2}-\nabla}{2 b_{1}}\right)}\right) \mathrm{d} E_{1}(\tau)=\nabla \mathrm{d} \tau$

where $\nabla=\sqrt{b_{2}{ }^{2}-4 b_{0} b_{1}}$.

Integrating both sides, we have

$$
\ln \left(\frac{E_{1}(\tau)-\frac{-b_{2}+\nabla}{2 b_{1}}}{E_{1}(\tau)-\frac{-b_{2}-\nabla}{2 b_{1}}}\right)=\tau \nabla+E_{0}
$$

Using boundary condition $E_{1}(\tau=0)=0$ we get

$$
E_{0}=\ln \left(\frac{-b_{2}+\nabla}{-b_{2}-\nabla}\right)
$$

Solving for $E_{1}(\tau)$, we obtain

$$
E_{1}(\tau)=\frac{\left(\mathrm{e}^{\tau \nabla}-1\right) \tilde{b}_{1} \tilde{b}_{2}}{2 b_{1}\left(\tilde{b}_{1}+\mathrm{e}^{\tau \nabla} \tilde{b}_{2}\right)}
$$

where $\tilde{b}_{1}=b_{2}+\nabla, \quad \tilde{b}_{2}=b_{2}-\nabla$.

In order to solve $B_{1}(\tau)$ explicitly, we substitute $C_{1}(\tau)$ and $E_{1}(\tau)$ in (B.5) and (B.6) into (B.4) .

$$
\begin{aligned}
B_{1}^{\prime}(\tau)= & \left(\frac{i u \alpha}{\beta}-\frac{i u \sigma_{r}^{2}}{\beta^{2}}\right)\left(1-\mathrm{e}^{-\beta \tau}\right)+\frac{i u \sigma_{r}^{2}}{\beta^{2}}\left(\mathrm{e}^{-\beta \tau}-\mathrm{e}^{-2 \beta \tau}\right) \\
& -\frac{\sigma_{r}^{2} u^{2}}{2 \beta^{2}}\left(1-2 \mathrm{e}^{-\beta \tau}+\mathrm{e}^{-2 \beta \tau}\right)+\frac{\gamma \tilde{b}_{1} \tilde{b}_{2}\left(\mathrm{e}^{\tau \nabla}-1\right)}{2 b_{1}\left(\tilde{b}_{1}+\mathrm{e}^{\tau \nabla} \tilde{b}_{2}\right)}
\end{aligned}
$$

Integrating with respect to $\tau$ and using boundary condition $B_{1}(\tau=0)=0$, then we get

$$
\begin{aligned}
B_{1}(\tau)= & \left(2 i u\left(\alpha \beta-\sigma_{r}^{2}\right)-\sigma_{r}^{2} u^{2}\right) \frac{\tau}{\beta^{2}} \\
& +\frac{i u \sigma_{r}^{2}}{2 \beta^{3}}\left(\mathrm{e}^{-\beta \tau}-1\right)^{2}+\frac{\sigma_{r}^{2} u^{2}}{4 \beta^{3}}\left(-4 \mathrm{e}^{-\beta \tau}+\mathrm{e}^{-2 \beta \tau}-3\right) \\
& +\left[\frac{-\tau \gamma \tilde{b}_{2}}{2 b_{1}}+\frac{\gamma\left(\tilde{b}_{1}+\tilde{b}_{2}\right)}{2 b_{1} \nabla} \ln \left(\frac{\tilde{b}_{1}+\mathrm{e}^{\tau \nabla} \tilde{b}_{2}}{\tilde{b}_{1}+\tilde{b}_{2}}\right)\right]
\end{aligned}
$$

The details of the proof for the characteristic function $f_{2}$ are similar to $f_{1}$.

Hence, we have

$$
\begin{aligned}
& f_{2}(t, x, r, v ; T-\tau, u) \\
= & \exp \left[i u x+B_{2}(\tau)+r C_{2}(\tau)+v E_{2}(\tau)\right]
\end{aligned}
$$

where $B_{2}(\tau), C_{2}(\tau)$ and $E_{2}(\tau)$ are as given in this Lemma.

We can thus evaluate the characteristic function in close form. However, we are interested in the probability $\tilde{P}_{j}$. These can be inverted from the characteristic functions by performing the following integration

$$
\begin{aligned}
& \tilde{P}_{j}(t, x, r, v ; T, \kappa) \\
= & \frac{1}{2}+\frac{1}{\pi} \int_{0+}^{\infty} \operatorname{Re}\left(\frac{\mathrm{e}^{\mathrm{iu \kappa}} f_{j}(t, x, v \cdot r ; T, u)}{i u}\right) \mathrm{d} u
\end{aligned}
$$

for $j=1,2$ where $X_{t}=\ln S_{t}$ and $\kappa=\ln K$, see Kendall et al. [10]. The proof is now complete. 